\title{
miR-9 functions as a tumor suppressor in ovarian serous carcinoma by targeting TLN1
}

\author{
HAOSHA TANG $^{1,2}$, LIANGQING YAO ${ }^{1}$, XIANG TAO $^{3}$, YI YU ${ }^{1,2}$, \\ MO CHEN $^{1,2}$, RONG ZHANG ${ }^{4}$ and CONGJIAN XU ${ }^{1,2,5,6}$
}

\author{
${ }^{1}$ Department of Obstetrics and Gynecology, Obstetrics and Gynecology Hospital, Fudan University; \\ ${ }^{2}$ Shanghai Key Laboratory of Female Reproductive Endocrine Related Diseases; ${ }^{3}$ Department of Pathology, \\ Obstetrics and Gynecology Hospital, Fudan University, Shanghai 200011; ${ }^{4}$ Department of Obstetrics and Gynecology, \\ Fengxian District Central Hospital, Shanghai 201400; ${ }^{5}$ Department of Obstetrics and Gynecology, Fudan University \\ School of Medicine; ${ }^{6}$ Institute of Biomedical Sciences, Fudan University, Shanghai 200032, P.R. China
}

Received March 4, 2013; Accepted May 20, 2013

DOI: $10.3892 /$ ijmm.2013.1400

\begin{abstract}
RNAs (miRNAs) are important regulators of gene expression during tumorigenesis. The downregulation of microRNA-9 (miR-9) has been reported in ovarian serous carcinoma (OSC), indicating a role for miR-9 in this type of cancer. In this study, we investigated the biological significance of miR-9 in OSC in vitro. Using 3 OSC cell lines, SKOV3, CAOV3 and OVCAR3, which underexpresss miR-9, we demonstrate that the exogenous miR-9 transfection inhibits OSC cell proliferation, migration and invasion. In addition, we demonstrate that the focal adhesion protein, talin 1 (TLN1), whose expression has been associated with OSC development and progression to metastasis, is a direct target of miR-9. TLN1 knockdown mimicked the effects of miR-9 overexpression. Moreover, the activation of the TLN1-modulated FAK/AKT pathway was inhibited by the increased miR-9 levels. These results suggest that miR-9 plays a role as a tumor suppressor in OSC by suppressing TLN1 expression.
\end{abstract}

\section{Introduction}

Epithelial ovarian cancer (EOC) is the sixth leading type cancer in women worldwide and the most lethal gynecological malignancy in the Western world (1). One of the greatest obstacles to improving outcome is the poor understanding of the molecular underpinnings of EOC pathogenesis and progression. MicroRNAs (miRNAs) are a large family of 21- to 23-nucleotide long, non-protein-coding RNAs that regulate gene expression by mediating target mRNA cleavage or translational inhibition (2). miRNAs participate in essential processes, such as cell differentiation, growth, apoptosis and invasion (3).

Correspondence to: Dr Congjian Xu, Department of Obstetrics and Gynecology, Obstetrics and Gynecology Hospital, Fudan University, 419 Fangxie Road, Shanghai 200011, P.R. China

E-mail: xucongjian@gmail.com

Key words: talin 1, microRNA-9, ovarian cancer
Evidence demonstrates that aberrant miRNA expression patterns exist in most, if not all human malignancies (4). A number of studies have shown that miRNA deregulation is of critical importance in EOC tumorigenesis (5), metastasis (6), recurrence (7) and chemoresistance (8).

Ovarian serous carcinoma (OSC) is the most common type of ovarian cancer, accounting for $68 \%$ of ovarian cancers and $88 \%$ of stage III and IV ovarian cancers (9). It has been reported that microRNA-9 (miR-9) is downregulated in OSC, as well as in endometrioid and clear cell ovarian carcinoma (5). Moreover, a further reduction in miR-9 levels has been found in recurrent OSC compared with the primary tumor (7). A recent study identified miRNAs with altered expression in a tumor-initiating subpopulation of OVCAR3 OSC cells, among which miR-9 was downregulated (10). Taken together, these data suggest that miR-9 acts as a tumor suppressor in OSC, although conflicting results have been shown in other types of cancer (11). To date, several targets of miR-9 associated with cancer have been identified: chromobox protein homolog 7 (CBX7) in human glioma (12), caudal type homeobox 2 (CDX2) in gastric cancer (8), nuclear factor (NF)- $\kappa \mathrm{B} 1$ in clear cell ovarian carcinoma (13), E-cadherin (11) and methylenetetrahydrofolate dehydrogenase ( $\mathrm{NADP}^{+}$dependent) 2, methenyltetrahydrofolate cyclohydrolase (MTHFD2) (14) in breast cancer. However, the precise role of miR-9 in cancer development and progression has not yet been elucidated.

In the present study, we investigated the functions of miR-9 in OSC cells and identified a novel target of miR-9. We demonstrate that miR-9 exerts its tumor suppressor activity by downregulating the expression of talin 1 (TLN1), a focal adhesion protein. TLN1 immunoprofiling in human OSC specimens revealed its overexpression in primary tumors compared with normal tissues, and an even higher expression in metastatic lesions compared with primary tumors.

\section{Materials and methods}

Clinical specimens and immunohistochemistry. Tissue microarrays (BC11115; US Biomax, Inc., Rockville, MD, USA) containing formalin-fixed, paraffin-embedded primary and 
lymph node metastatic OSC tissues, normal ovarian tissue and tumor-adjacent ovarian tissues were subjected to immunoprofiling for TLN1 expression. In addition, OSC tissues were obtained at Shanghai Fengxian District Central Hospital between 2008 and 2011 with informed patient consent for TLN1 immunostaining. The study was approved by the Ethics Committee of Shanghai Fengxian District Central Hospital. H\&E-stained sections of primary OSC tissues were reviewed by an experienced gynecological pathologist to grade the tumors using a two-tier system (15). In total, 14 normal ovarian tissues, 13 tumor-adjacent ovarian tissues, 67 primary (18 low- and 39 highgrade) lesions and 26 metastatic lesions of OSC were evaluated for TLN1 expression. Immunostaining was performed using an EnVision $^{\mathrm{TM}}+$ System peroxidase kit (Dako, Glostrup, Denmark) and a monoclonal mouse antibody against TLN1 (1:200 dilution; Santa Cruz Biotechnology, Inc., Santa Cruz, CA, USA). Fromowitz's standard was used to semiquantitatively assess the staining intensity as previously described (16). Briefly, the final scoring of TLN1 staining was judged by the positive range score plus the positive extent score, so that the TLN1 level was analyzed comprehensively by the extent and range of staining.

Cell lines. The human OSC cell lines, SKOV3, CAOV3 and OVCAR3, were purchased from the Cell Bank of the Chinese Academy of Sciences (Shanghai, China). SKOV3 cells were maintained in Roswell Park Memorial Institute (RPMI)-1640 medium (Invitrogen, Carslbad, CA, USA) supplemented with $10 \%$ fetal bovine serum (FBS). OVCAR3 cells were maintained in RPMI-1640 (Invitrogen) supplemented with $20 \%$ FBS. CAOV3 cells were maintained in Dulbecco's modified Eagle's medium in high glucose (Invitrogen) supplemented with $10 \%$ FBS. HOSEpiC human ovarian surface epithelial cells were purchased from ScienCell Research Laboratories (Carlsbad, CA, USA) and cultured in ovarian epithelial cell medium (ScienCell Research Laboratories).

Transient transfection of miRNA and siRNA. The overexpression or knockdown of miR-9 was carried out by transfecting the OSC cells (SKOV3, CAOV3 and OVCAR3) with $100 \mathrm{nM}$ miR-9 mimic or $50 \mathrm{nM}$ antagomir-9 (an anti-miR-9 molecule) using Lipofectamine 2000 reagent following the manufacturer's instructions. In order to inhibit TLN1 expression in the OSC cells, specific siRNA for TLN1 was similarly transfected into the OSC cells at a concentration of $100 \mathrm{nM}$. Forty-eight hours after transfection, western blot analysis and/or quantitative reverse transcription-PCR (qRT-PCR) were conducted to confirm the transfection efficiencies. The miRNAs and siRNAs (including respective negative controls) were all purchased from RiboBio Co., Ltd., Guangzhou, China.

Vector construction and luciferase reporter assay. The fulllength 3' untranslated region (3'UTR) of TLN1 (454 bp) was amplified from the genomic DNA of SKOV3 cells by PCR (forward primer, 5'-CGAGCTCGATAGAAGAAGCCTCTTCT ATTT-3'; reverse primer, 5'-ACGCGTCGACTCTAGATTGTA GGTAGAATCAT-3') and cloned downstream of the firefly luciferase gene into the pmirGLO dual-luciferase miRNA target expression vector (pmirGLO Vector; Promega, Madison, WI, USA) at the SacI and SalI sites. The fragment of the TLN1-3'UTR mutant (TLN1-3'UTRmu) construct, which contained a mutational miR-9 binding site, was generated from the TLN1-3'UTR construct using the QuickChange ${ }^{\text {TM }}$ SiteDirected Mutagenesis kit (Agilent Technologies, Inc., Santa Clara, CA, USA). The TLN1-3'UTR or TLN1-3'UTRmu construct $(100 \mathrm{ng})$ was co-transfected with $\mathrm{miR}-9$ or the negative control $(50 \mathrm{nM})$ into the OSC cells for luciferase reporter assays. Twenty-four hours after transfection, cells were collected for Luciferase activity detection using the Dual-Luciferase Reporter Assay System (Promega).

$q R T-P C R$. To determine the relative level of miR-9 or TLN1 expression, total cellular RNA from the OSC cells was extracted using TRIzol reagent (Invitrogen). cDNA was then synthesized with a miRNA-specific stem-loop primer or oligo(dT) using the Script ${ }^{\mathrm{TM}}$ First-Strand Synthesis System for RT-PCR (Invitrogen). Subsequently, qRT-PCR was performed with SYBR-Green PCR master mix (Applied Biosystems, Inc. Foster City, CA, USA) on an ABI 7500 System. The U6 or glyceraldehyde 3-phosphate dehydrogenase (GAPDH) gene was used as an endogenous control. RT primers were as follows: miR-9 RT, 5'-TCGTATCCAGTGCAGGGTCCGAGG TGCACTGGATACGACTCATACAG-3'; U6RT,5'-GTCGTAT CCAGTGCAGGGTCCGAGGTATTCGCACTGGATACGAC AAAATATGGAAC-3'; TLN1/GAPDH RT, oligo(dT). PCR primers were: miR-9 forward, 5'-GCCCGCTCTTTGGTTAT CTAG-3'; U6 forward, 5-TGCGGGTGCTCGCTTCGGC AGC-3'; miR-9/U6 reverse, 5'-CCAGTGCAGGGTCCG AGGT-3. TLN1 forward, 5'-AGTGACGGACAGCATCAA CCAG-3', TLN1 reverse, 5'-GGATTCTCCAGGAGTTCTC GGA-3'. GAPDH forward, 5'-ACCCACTCCTCCACCT TTG-3', GAPDH reverse, 5'-CACCACCCTGTTGCTG TAG-3'. The relative gene expression between multiple samples was quantified by normalization against endogenous U6 or GAPDH using the $\Delta \mathrm{Ct}$ method. Fold-changes were calculated as $2^{-\Delta \Delta C(t)}$.

Western blot analysis. Total proteins from the OSC cells were extracted using RIPA lysis buffer (Beyotime Biotech, Haimen, China). Proteins were quantified (bicinchoninic acid assay; Bio-Rad), and separated on 6\% SDS-PAGE gels and subsequently transferred onto nitrocellulose membranes. The membranes were then blocked in $5 \%$ bovine serum albumin (BSA) for $1 \mathrm{~h}$ at room temperature, followed by incubation with each primary antibody overnight at $4^{\circ} \mathrm{C}$. The following primary antibodies were used: rabbit or mouse monoclonal antibodies to TLN1, FAK, phospho-FAK (Y397), AKT, phospho-Akt (S473) and GAPDH (Abcam, Cambridge, MA, USA). The membranes were then incubated with species-specific horseradish peroxidase-labeled secondary antibodies. Immunoreactive proteins were visualized using enhanced chemiluminescence detection system (Amersham/GE Healthcare, San Diego, CA, USA). Protein levels were normalized to GAPDH expression.

Transwell migration and invasion assays. Forty-eight hours after transfection, $1 \times 10^{5}$ OSC cells suspended in $100 \mu 10.1 \%$ FBS medium were added to the upper chamber of a Transwell chamber ( $8 \mu \mathrm{m}$ pore size, $6.5 \mathrm{~mm}$ in diameter; Corning Inc., Corning, NY, USA). For invasion assay, the filter membrane was coated with Matrigel (BD Biosciences, Franklin Lakes, NJ, USA) prior to the experiment. A total of $600 \mu 1$ of $10 \%$ FBS 


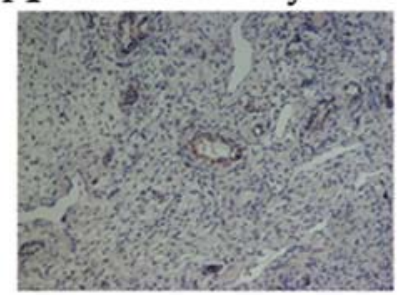

primary tumor

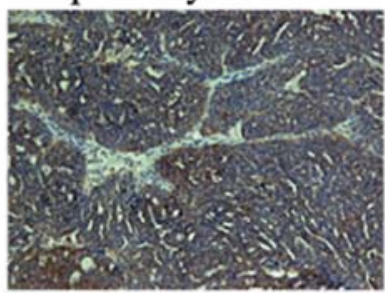

\section{B low-grade tumor}

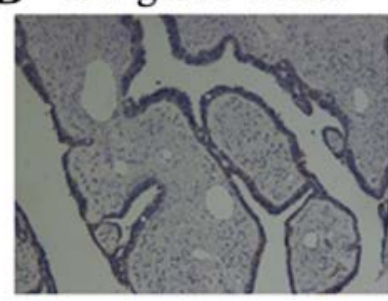

A normal ovary tumor adjacent tissue

lymph node metastasis

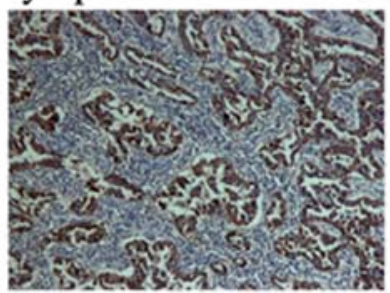

high-grade tumor

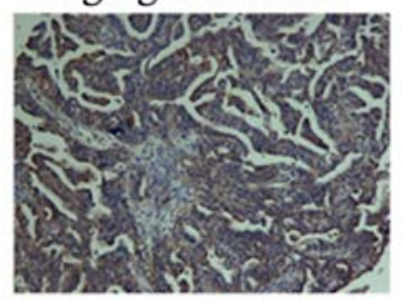

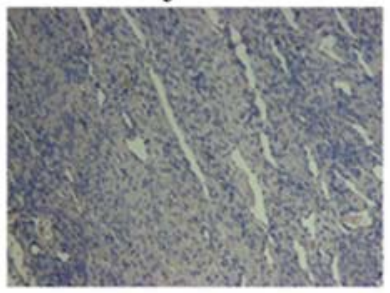

Figure 1. Talin 1 (TLN1) immunoreactivity was analyzed in ovarian serous carcinoma (OSC) samples. (A) TLN1 expression in normal ovarian tissue, tumor-adjacent tissue, primary OSC and lymph node metastatic lesion of OSC. (B) TLN1 expression in low- and high-grade OSC.

medium was added to the lower chamber. After incubation for $18 \mathrm{~h}$, the migrated/invaded cells were fixed, stained with $0.5 \%$ crystal violet and counted under an inverted microscope.

MTT and colony formation assays. The OSC cells were transfected with miRNA or siRNA as described above, and then seeded into 96 -well plates at $2 \times 10^{3}$ cells/well. The numbers of viable cells were determined by MTT assay at 1, 2, 3, 4 and 5 days after transfection.

For colony formation assay, cells were seeded into $35-\mathrm{mm}$ culture dishes at 200 cells/dish $24 \mathrm{~h}$ after transfection. The medium was replaced with fresh culture medium every 3-4 days. When the cells formed visible colonies (after approximately 2 weeks), the cells were stained with crystal violet and colonies containing $>50$ cells were counted.

Statistical analysis. One-way ANOVA or the Student's t-test was performed using SPSS version 17.0 software to determine the statistical significance between groups. A P-value $<0.05$ was considered to indicate a statistically significant difference. Data are presented as the means \pm standard deviation (SD) of measurement.

\section{Results}

TLN1 overexpression correlates with OSC development and progression. Immunohistochemical staining was performed to
Table I. Expression of TLN1 in human ovarian serous carcinoma.

A, Quantitative analysis of TLN1 expression in human ovarian tissue

\begin{tabular}{lccc}
\hline Specimen & $\mathrm{n}$ & $\begin{array}{c}\text { Score } \\
(\text { mean } \pm \text { SD) }\end{array}$ & P-value \\
\hline Normal & 14 & $0.43 \pm 0.514$ & \\
Adjacent & 13 & $0.46 \pm 0.660$ & 0.886 \\
Primary tumor & 67 & $3.54 \pm 1.869$ & $<0.001^{\mathrm{a}}$ \\
Metastasis & 26 & $4.50 \pm 1.530$ & $<0.001^{\mathrm{a}} ; 0.022^{\mathrm{b}, \mathrm{c}}$ \\
\hline
\end{tabular}

TLN1 expression was evaluated by Fromowitz's standard. Specimens included normal ovarian tissue, normal tissue adjacent to the tumor foci, primary ovarian serous carcinoma lesion and lymph node metastatic lesion. Statistical difference at ${ }^{\mathrm{a}} \mathrm{P}<0.01$ and ${ }^{\mathrm{b}} \mathrm{P}<0.05$, compared with normal ovarian tissue, unless otherwise indicated. ${ }^{\mathrm{c} S t a t i s t i c a l}$ difference compared with primary tumor tissue.

$\mathrm{B}$, TLN1 expression in primary tumor

\begin{tabular}{|c|c|c|c|}
\hline Tumor grade & $\mathrm{n}$ & $\begin{array}{c}\text { Score } \\
(\text { mean } \pm \mathrm{SD})\end{array}$ & P-value \\
\hline Low & 18 & $1.67 \pm 1.237$ & \\
\hline High & 49 & $4.20 \pm 1.554$ & $<0.001^{\mathrm{a}}$ \\
\hline
\end{tabular}

TLN1 expression was evaluated by Fromowitz's standard. Comparative TLN1 levels in primary ovarian serous carcinoma. Statistical difference at ${ }^{a} \mathrm{P}<0.01$, compared with low-grade tumor. TLN1, talin 1.

determine the significance of TLN1 expression in human OSC. A representative image of normal ovarian tissue, tumor-adjacent ovarian tissue, primary OSC tissue and a metastatic tumor to the lymph node is shown in Fig. 1A. Cytoplasmic TLN1 immunoreactivity was comparably weak or undetectable in the normal and tumor-adjacent ovarian tissues $(\mathrm{P}=0.886)$ (Table I). By contrast, TLN1 expression was markedly elevated in the primary OSC tissue and lymph node metastatic lesions compared with normal ovarian tissue $(\mathrm{P}<0.001, \mathrm{P}<0.001$, respectively) (Table I). TLN1 expression in the metastatic lesions was significantly higher compared with that in primary tumors $(\mathrm{P}=0.022)$ (Table I). In addition, TLN1 was differentially expressed between low- and high-grade OSC (Fig. 1B), i.e., the intensity of TLN1 immunoreactivity was significantly higher in the high- compared with the low-grade tumors $(\mathrm{P}<0.001)$ (Table I).

miR-9 inhibits OSC cell growth. Quantitative analysis of mature miR-9 expression levels in the OSC cell lines, SKOV3, CAOV3 and OVCAR3, and the normal ovarian surface epithelial cell line, HOSEpiC, revealed lower miR-9 levels in the OSC cells compared with the normal ovarian surface epithelial cells (Fig. 2A). The effect of miR-9 overexpression on OSC cell growth was investigated. Twenty-four hours after miRNA transfection, miR-9 levels were determined by qRT-PCR to confirm the transfection efficiencies (Fig. 2B). MTT assay revealed that miR-9 suppressed OSC cell growth in a time-dependant manner (Fig. 2C-E). Although there was no significant difference in cell 
A

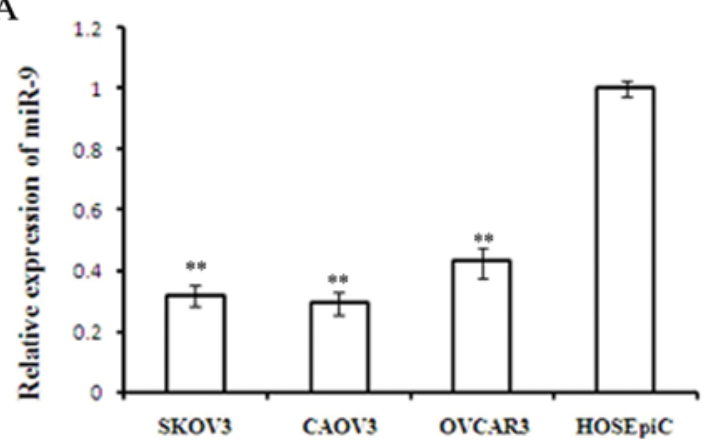

SKOV3

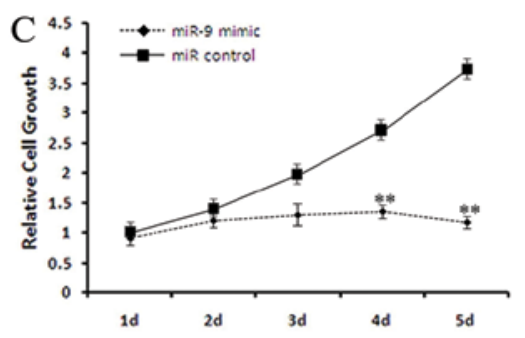

$\mathrm{F}$

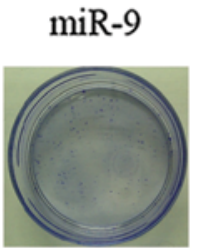

miR control

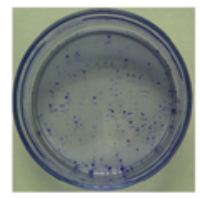

SKOV3

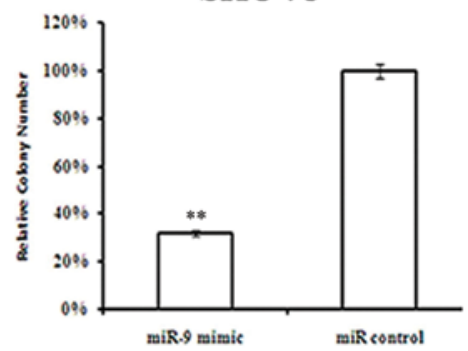

B

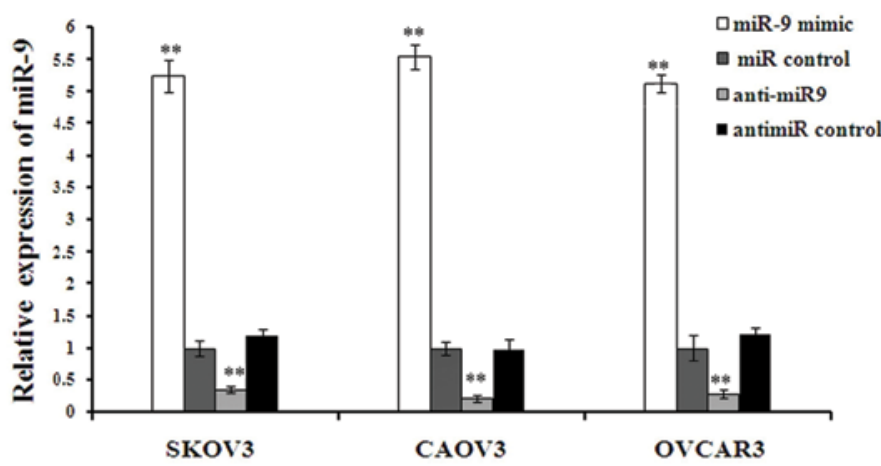

CAOV3

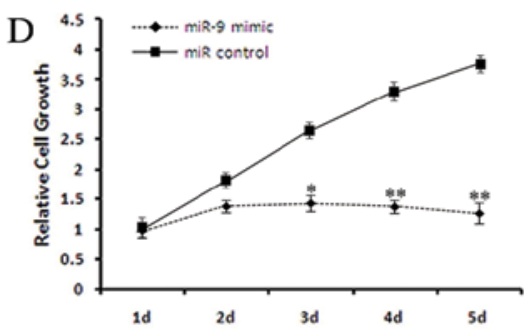

G

miR-9

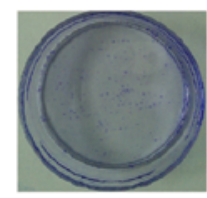

miR control

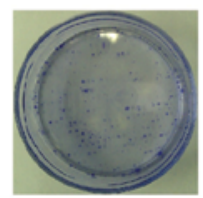

CAOV3

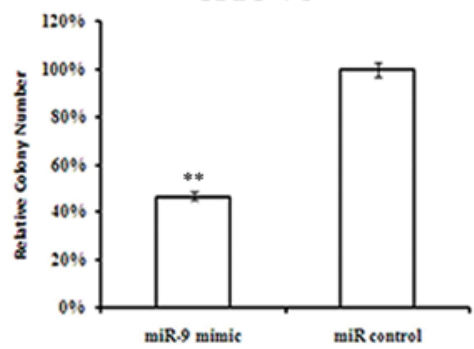

OVCAR3

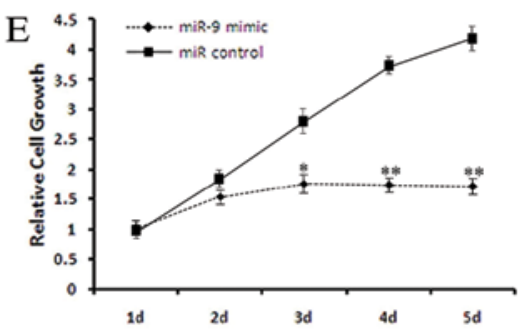

H $\quad \operatorname{miR}-9$

miR control
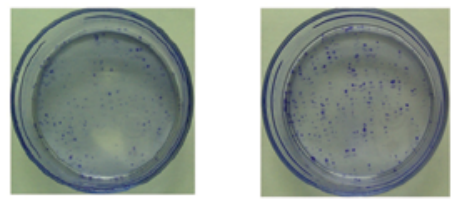

OVCAR3

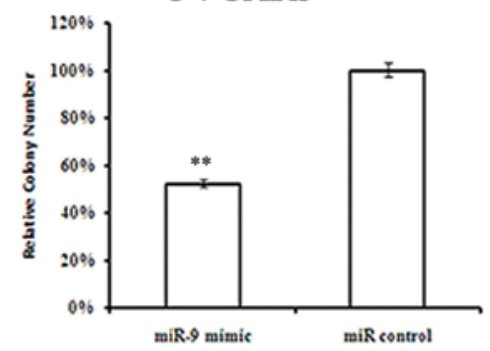

Figure 2. Overexpression of miR-9 suppresses ovarian serous carcinoma (OSC) cell growth. (A) Quantitative RT-PCR (qRT-PCR) analysis of miR-9 in the OSC cell lines, SKOV3, CAOV3 and OVCAR3, and the normal ovarian surface epithelial cell line, HOSEpiC, represented as normalized relative expression. (B) Levels of miR-9 in OSC cells analyzed $48 \mathrm{~h}$ after miRNA transfection. (C-E) Cell viability was measured by MTT assay on days 1-5 after miR-9 mimic or control transfection in SKOV3, CAOV3 and OVCAR3 cells, respectively. (F-H) Cell proliferation was measured by colony formation assay after miR-9 mimic or control transfection in SKOV3, CAOV3 and OVCAR3 cells, respectively. $\left(\mathrm{n}=3 ;{ }^{*} \mathrm{P}<0.05,{ }^{* *} \mathrm{P}<0.01\right)$.

growth between the miR-9-transfected cells and those in the control groups during the first 2 days, miR- 9 overexpression significantly inhibited cell growth at days 3 and 4 post-transfection. By day 5, the growth of SKOV3, CAOV3 and OVCAR3 cells was reduced by $73.5(\mathrm{P}<0.01), 71.6(\mathrm{P}<0.01)$ and $61.2 \%$ $(\mathrm{P}<0.01)$, respectively. To further validate the anti-proliferative effects of miR-9 on OSC cells, a colony formation assay was performed. Less colony formation was observed in the miR9-transfected cells compared with the control-transfected cells (Fig. 2F-H), indicating that miR-9 suppressed the colony forming ability of OSC cells.
miR-9 impairs SKOV3, CAOV3 and OVCAR3 cell migration and invasion. Previous studies have demonstrated that miR-9 inhibits the migration and invasion of hepatocellular carcinoma (HCC) cells and uveal melanoma cells $(17,18)$. We wished to determine whether miR-9 affects the migratory and invasive capacity of OSC cells. As miR-9 overexpression did not cause a significant difference in OSC cell proliferation until $72 \mathrm{~h}$ after transfection, we performed Transwell migration and invasion assays at $48 \mathrm{~h}$ after miRNA transfection. The migratory and invasive ability of the OSC cells was significantly impaired by miR-9 overexpression (Fig. 3A). 
A
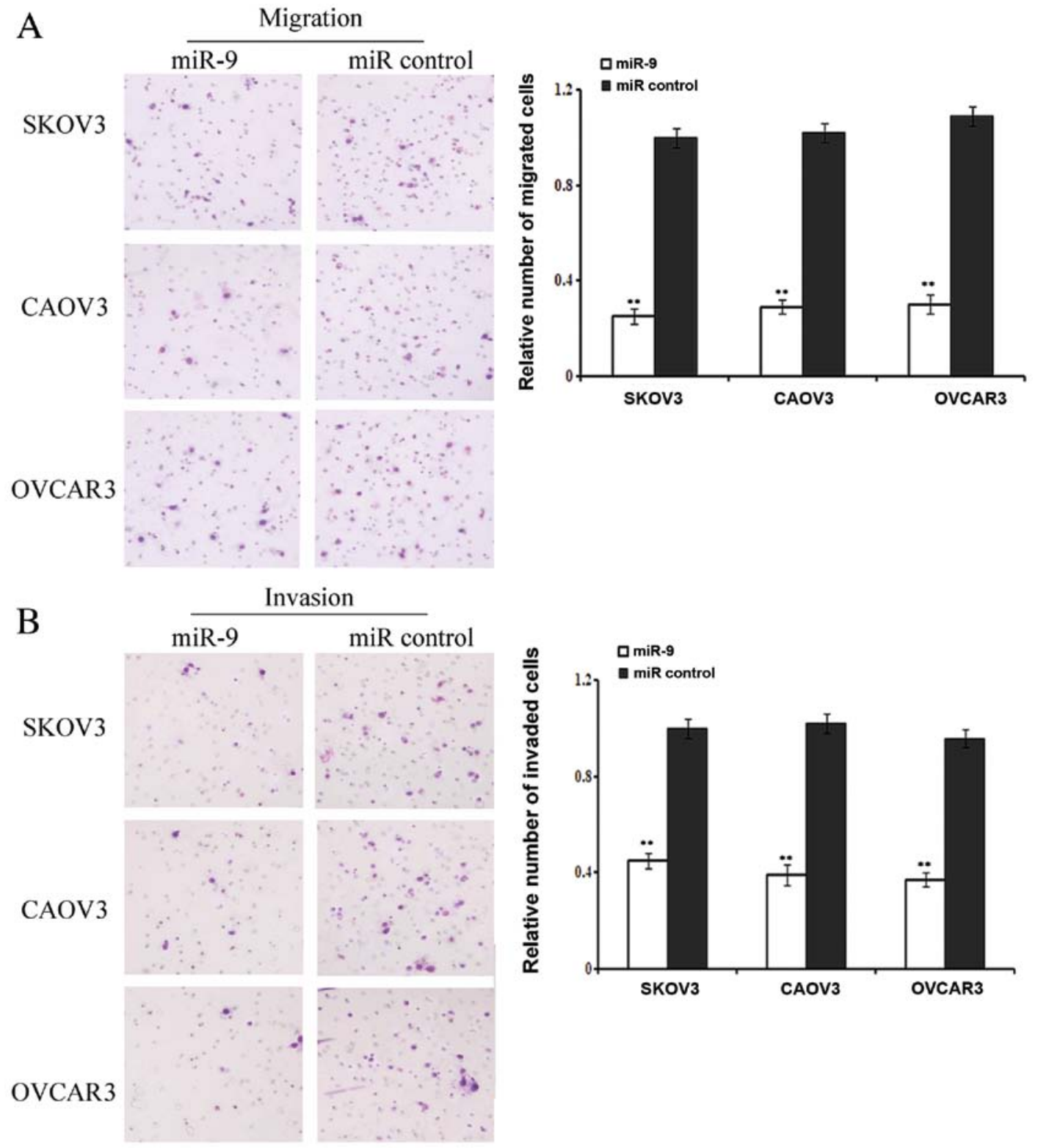

Figure 3. Overexpression of miR-9 inhibits cell migration and invasion. (A) Representative micrographs of cell migration assay (left panel) and quantification (right panel) from 3 independent experiments. (B) Representative micrographs of cell invasion assay (left panel) and quantification (right panel) from 3 independent experiments $\left(\mathrm{n}=3 ;{ }^{* *} \mathrm{P}<0.01\right)$.

miR-9 suppresses TLN1 mRNA and protein expression by binding to its 3'UTR. Using miRecords (http://mirecords.biolead. org), TargetScan (http://www.targetscan.org), PicTar (http:// www.ncRNA.org/) and miRanda (http://www.microrna.org), we predicted that TLN1 is a candidate target gene of miR-9 since it carries a putative miR-9 binding site within its 3'UTR. To confirm that miR-9 binds to this region and induces translational repression, we constructed 2 TLN1 3'UTR luciferase reporter vectors bearing either a wild-type or a mutated sequence of the predicted miR-9 binding site (Fig. 4A). Co-transfection experiments revealed that exogenous miR-9 decreased the luciferase activity of TLN1-3'UTR $(\mathrm{P}<0.01)$, but had little effect on TLN1-3'UTRmu (Fig. 4B), indicating that miR-9 directly interacts with the predicted target site in TLN1-3'UTR. To further confirm that miR-9 plays a functional role in TLN1 downregulation, OSC cells were transfected with miR-9 mimic or anti-miR-9. Subsequent RT-PCR and western blot analysis indicated that miR-9 overexpression led to a decline in TLN1 mRNA and protein levels (Fig. 4C); by contrast, the knockdown of endogenous miR-9 resulted in an increase in TLN1 mRNA and protein expression (Fig. 4D). These findings suggest that the miR-9-mediated suppression of TLN1 expression may be associated with mRNA degradation.

Inhibition of TLN1 expression mimics the anti-proliferative, anti-migratory and anti-invasive effects of miR-9 overexpression on OSC cells. As we found that TLN1 was upregulated in OSC and correlated with OSC aggressiveness and metastasis, and that miR-9 directly suppressed the expression of TLN1, we wished to determine whether a reduction in TLN1 expression may provide an explanation for the observed effects of miR-9 overexpression. TLN1 knockdown with siRNA transfection was performed, followed by MTT, Transwell migration and invasion assays. The successful knockdown of TLN1 in OSC 
A

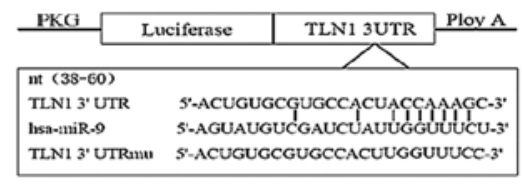

B

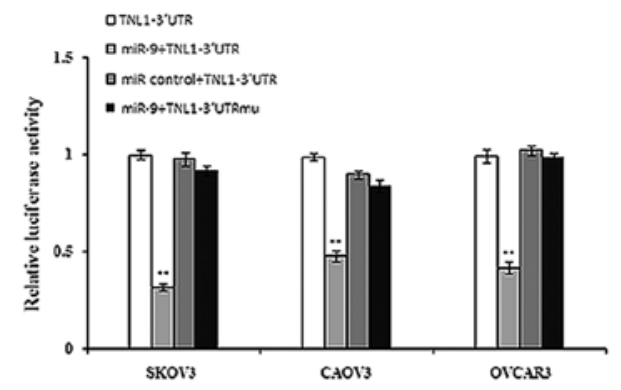

C
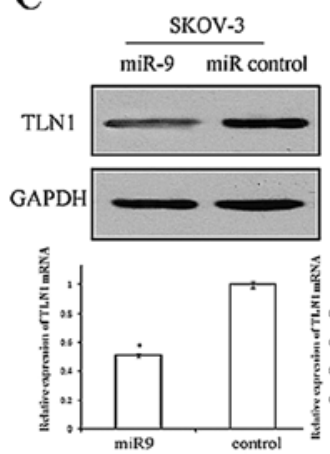
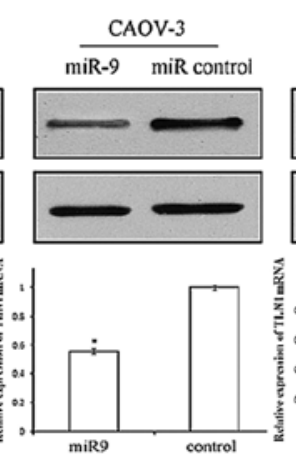
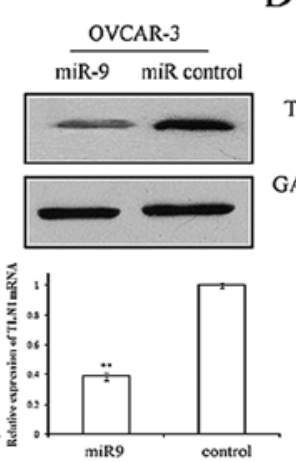

D
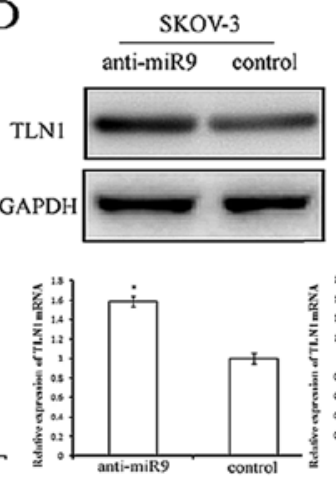
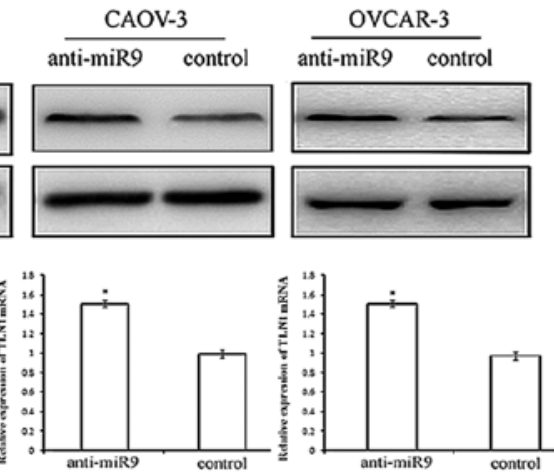

Figure 4. Talin 1 (TLN1) is a direct target of miR-9 in ovarian serous carcinoma (OSC) cells. (A) Construct of the luciferase-TLN1-3'-untranslated region (3'UTR) reporter vector. (B) Luciferase activity was measured $24 \mathrm{~h}$ after co-transfection of miRNA and wild-type or mutant TLN1-3'UTR into the OSC cells. Cells tranfected with only wild-type TLN1-3'UTR were used as the controls. (C and D) Representative westen blot of TLN1 protein (top panel) and relative TLN1 mRNA levels determined by quantitative RT-PCR (qRT-PCR) (bottom panel) in miR-9- or anti-miR-9-transfected OSC cells, respectively. (n=3; ${ }^{* *}$ < 0.01 ).

cells was confirmed by qRT-PCR and western blot analysis (Fig. 5A). As with miR-9 overexpression, the decrease in TLN1 expression significantly inhibited OSC cell growth, migration and invasion (Fig. 5B-D). These results indicate that TLN1 is involved in the miR-9-mediated suppression of OSC, and TLN1 inhibition may explain, at least in part, the anti-proliferative, anti-migratory and anti-invasive effects of miR-9 on OSC cells.

mir-9 inhibits the activation of the FAK/AKT signaling pathway. Since we demonstrated that TLN1 is a direct and functional target of miR-9, we further investigated the effects of miR-9 overexpression on the TLN1-regulated FAK/AKT signaling pathway (19). Forty-eight hours after tranfeciton with miR-9 or miR control, the OSC cells were subjected to western blot analysis to evaluate the total and phosphorylated protein levels of FAK and AKT. miR-9 overexpression in the OSC cells resulted in a marked reduction in the phosphorylation levels of FAK and AKT, suggesting the inhibtion of the FAK/AKT pathway (Fig. 6).

\section{Discussion}

miRNAs have emerged as a novel pathway of tumorigenesis (20). Evidence shows that miRNAs function as either tumor suppressor or oncogenes. Recent studies have demonstrate that miR-9 is downregulated by aberrant $\mathrm{CpG}$ island methylation in a variety of human cancers, including breast (21), gastric (22) and lung cancer (19). Moreover, evidence suggests that miR-9 downregulation is associated with cancer metastasis (23) and the 'cancer stem cell' phenotype $(10,24)$. miR-9 is downregu- lated in human ovarian serous, endometrioid and clear cell carcinoma (5). In addition, a previous study demonstrated that the miR-9 expression level was significantly lower in recurrent OSC compared with primary tumors (7). Therefore, miR-9 may act as a tumor suppressor in OSC, and its exact role in OSC biology needs to be further elucidated.

In this study, we first demonstrated that endogenous miR-9 levels are lower in OSC cells compared with normal ovarian surface epithelial cells (HOSEpiC), consistent with previous reports of lower miR-9 levels in OSC tissues (5). We then used a gain-of-function approach by transfecting miR-9 mimics into OSC cells to determine the function of miR-9. Changes in cell proliferation are key phenotypes observed in malignant transformation; uncontrolled migration and invasion lead to metastasis, which causes the human cancer mortality rate to reach as high as $90 \%(25,26)$. Our results revealed that the overexpression of miR-9 inhibited OSC cell proliferation, migration and invasion, supporting a tumor suppressor-like role for miR-9 in OSC development and progression.

A few biological targets of miR-9 have been identified. For example, miR-9 downregulates MTHFD2 expression, resulting in the inhibition of cell proliferation in breast cancer (14); miR-9 suppresses uveal melanoma cell migration and invasion by targeting $\mathrm{NF}-\kappa \mathrm{B} 1$ (18). In this study, we demonstrate that miR-9 inhibits TLN1 expression by targeting its 3'UTR, indicating that TLN1 is a direct target of miR-9. Furthermore, the knockdown of TLN1 expression mimicked the anti-proliferative, anti-migratory and anti-invasive effects of miR-9 overexpression on OSC cells. Therefore, TLN1 may be one of the key mediators of OSC suppression by miR-9. 

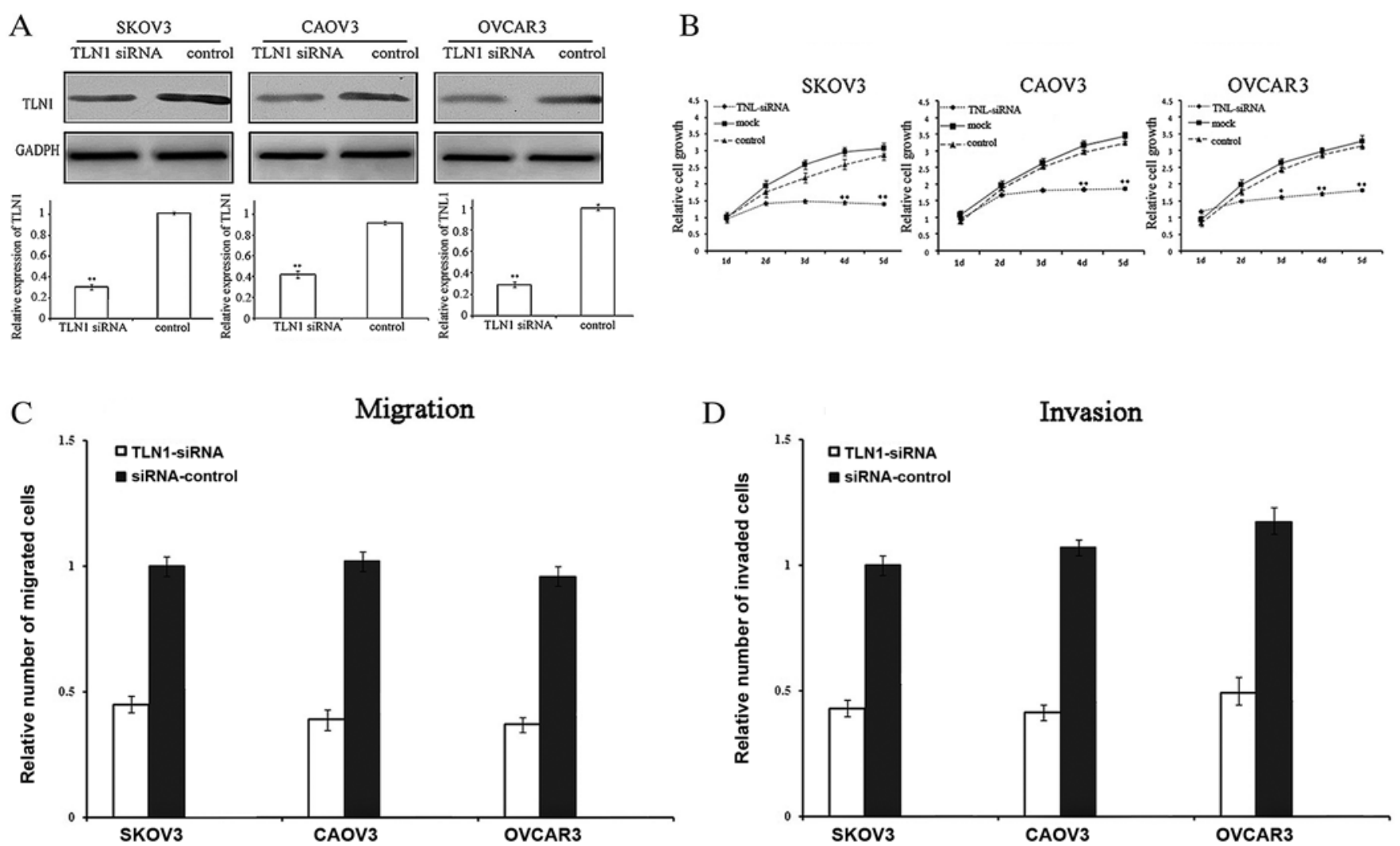

Figure 5. Knockdown of talin 1 (TLN1) reduces the growth, migratory and invasive ability of ovarian serous carcinoma (OSC) cells. OSC cells were transfected with TLN1-siRNA or siRNA control. (A) TLN1 protein (top panel) or mRNA expression (bottom panel) were detected by western blot analysis or quantitative RT-PCR (qRT-PCR), respectively at $48 \mathrm{~h}$ post-transfection with siRNAs. (B-D) Cell growth, migration and invasion were measured at $24 \mathrm{~h}$ (cell growth) or $48 \mathrm{~h}$ (cell migration and invasion) after transfection $\left(\mathrm{n}=3 ;{ }^{*} \mathrm{P}<0.05,{ }^{* * *} \mathrm{P}<0.01\right)$.
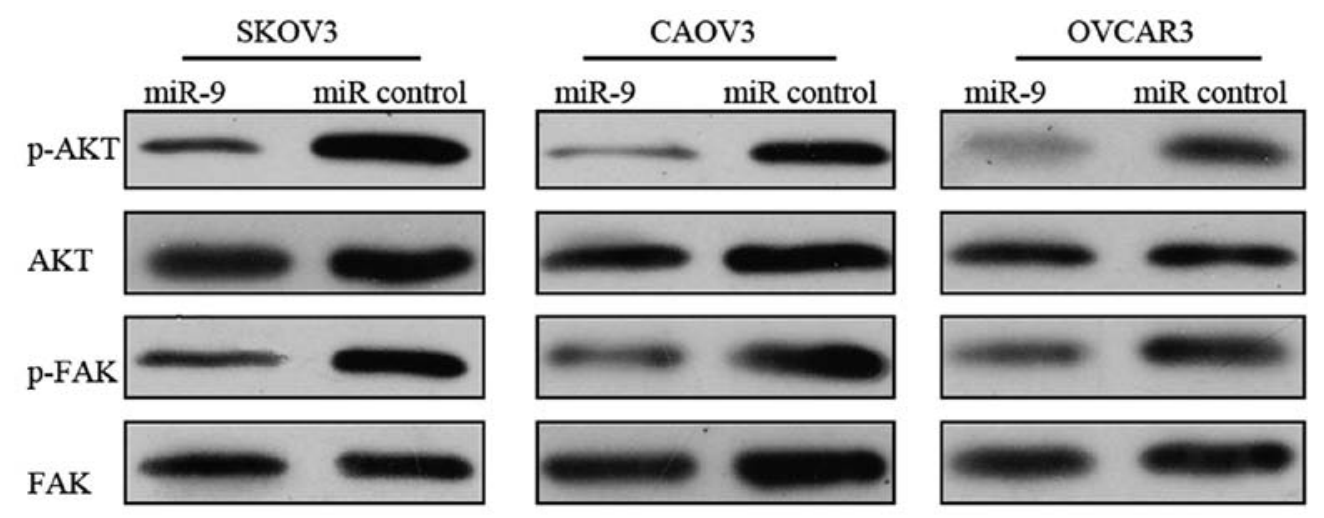

Figure 6. Overexpression of miR-9 inhibits the activation of the FAK/AKT pathway. Total and phosphorylated FAK and AKT were detected $48 \mathrm{~h}$ posttransfection of miR-9 in ovarian serous carcinoma (OSC) cells. Representative image from western blot analysis is shown.

Recent studies have suggested that TLN1 is involved in the progression of cancer (27-29). Our previous study demonstrated that serum TLN1 was upregulated during the early and advanced phase of a rat model of induced OSC, suggesting a role for TLN1 in the onset and progression of this malignancy (30). Consistent with these observations, the present study revealed a higher expression of TLN1 in OSC tissues as compared with tumor-adjacent normal ovarian tissues. In addition, the TLN1 expression level was significantly higher in the lymph node metastatic lesions compared with primary tumors, suggesting the involvement of TLN1 in the metastatic process. As evidence shows that there are 2 distinct types of OSC, namely, low- and high-grade OSC, which exhibit different pathogenesis and clinicopathological features (15), we analyzed TLN1 expression after grading the primary OSC specimens using the two-tier system. Of note, the high-grade OSC samples exhibited a significantly higher TLN1 expression compared with the lowgrade OSC samples. Thus, TLN1 expression in OSC may be used as a promising predictor of aggressiveness, as high-grade cancer is associated with more aggressive behavior and is an independent predictor of poor survival (31). Given that TLN1 is a target gene of miR-9, the suppression of miR-9 expression 
may account for the overexpression of TLN1 in OSC, although the involvement of other mechanisms cannot be excluded.

TLN1 is a component of the focal adhesion complex that is responsible for mediating the interaction between the actin cytoskeleton and integrins. In addition to its structural role, TLN1 plays an essential role in integrin activation (32). TLN1-mediated integrin activation is key to focal adhesion signaling and the induction of downstream pathways that ultimately regulate adhesion, proliferation, anoikis, survival and tumor progression $(27,28)$. Previous studies have indicated that the activation of the FAK/AKT pathway is associated with increased proliferation, migration and invasion in a variety of tumors (29,33-35). TLN1 overexpression in prostate cancer cells has been shown to result in a significant enhancement in the phosphorylation of FAK and AKT (36). In the present study, miR-9 overexpression led to a marked reduction in FAK and AKT phosphorylation levels. The miR-9-mediated suppression of TLN1 expression may provide an explanation for the observed inhibition of the FAK/AKT pathway; however, since miR-9 regulates multiple gene expressions, the involvement of other mechanisms cannot be excluded. The fact that miR-9 inhibits the activation of the FAK/AKT signaling pathway, and that NF- $\kappa \mathrm{B} 1$ is a downstream effector of the AKT survival pathway, has led to the hypothesis that the inhibition of the FAK/AKT pathway may be the mechanism through which miR-9 targets NF- $\kappa \mathrm{B} 1$ expression in certain tumors $(13,18)$.

In conclusion, miR-9 is downregulated in OSC cells compared with normal ovarian surface epithelial cells. miR-9 acts as a tumor suppressor by inhibiting OSC cell growth, migration and invasion. A direct and functional target of miR-9, TLN1, is overexpressed and associated with aggressiveness and metastasis in OSC. Our results indicate that the downregulation of miR-9 in OSC may contribute to the malignant phenotype by maintaining a high level of TLN1. Thus, the elucidation of the roles of miR-9 and its target gene, TLN1, in OSC may aid in the development of promising strategies for the targeted therapy of OSC in the future.

\section{References}

1. Cannistra SA: Cancer of the ovary. N Engl J Med 351: 2519-2529, 2004.

2. Mo YY: MicroRNA regulatory networks and human disease. Cell Mol Life Sci 69: 3529-3531, 2012.

3. Zamore PD and Haley B: Ribo-gnome: the big world of small RNAs. Science 309: 1519-1524, 2005.

4. Lu J, Getz G, Miska EA, et al: MicroRNA expression profiles classify human cancers. Nature 435: 834-838, 2005.

5. Iorio MV, Visone R, Di Leva G, et al: MicroRNA signatures in human ovarian cancer. Cancer Res 67: 8699-8707, 2007.

6. Olson P, Lu J, Zhang H, et al: MicroRNA dynamics in the stages of tumorigenesis correlate with hallmark capabilities of cancer. Genes Dev 23: 2152-2165, 2009.

7. Laios A, O'Toole S, Flavin R, et al: Potential role of miR-9 and miR-223 in recurrent ovarian cancer. Mol Cancer 7: 35, 2008.

8. Rotkrua P, Akiyama Y, Hashimoto Y, Otsubo T and Yuasa Y: MiR-9 downregulates CDX2 expression in gastric cancer cells. Int J Cancer 129: 2611-2620, 2011.

9. Moore RG, Chung M, Granai CO, Gajewski W and Steinhoff MM: Incidence of metastasis to the ovaries from nongenital tract primary tumors. Gynecol Oncol 93: 87-91, 2004.

10. Guo R, Wu Q, Liu F and Wang Y: Description of the CD133+ subpopulation of the human ovarian cancer cell line OVCAR3. Oncol Rep 25: 141-146, 2011.

11. Ma L, Young J,Prabhala H, et al: $\mathrm{miR}-9$, a MYC/MYCN-activated microRNA, regulates E-cadherin and cancer metastasis. Nat Cell Biol 12: 247-256, 2010.
12. Chao TF, Zhang Y, Yan XQ, et al: MiR-9 regulates the expression of CBX7 in human glioma. Zhongguo Yi Xue Ke Xue Yuan Xue Bao 30: 268-274, 2008 (In Chinese).

13. Guo LM, Pu Y, Han Z, et al: MicroRNA-9 inhibits ovarian cancer cell growth through regulation of NF-kappaB1. FEBS J 276: 5537-5546, 2009.

14. Selcuklu SD, Donoghue MT, Rehmet K, et al: MicroRNA-9 inhibition of cell proliferation and identification of novel miR-9 targets by transcriptome profiling in breast cancer cells. J Biol Chem 287: 29516-29528, 2012.

15. Malpica A, Deavers MT, Lu K, et al: Grading ovarian serous carcinoma using a two-tier system. Am J Surg Pathol 28: 496-504, 2004.

16. Kobel M, Kalloger SE, Huntsman DG, et al: Differences in tumor type in low-stage versus high-stage ovarian carcinomas. Int J Gynecol Pathol 29: 203-211, 2010.

17. Tan HX, Wang Q, Chen LZ, et al: MicroRNA-9 reduces cell invasion and E-cadherin secretion in SK-Hep-1 cell. Med Oncol 27: 654-660, 2010

18. Liu N, Sun Q, Chen J, et al: MicroRNA-9 suppresses uveal melanoma cell migration and invasion through the NF- $\kappa \mathrm{B} 1$ pathway. Oncol Rep 28: 961-968, 2012.

19. Heller G, Weinzierl M, Noll C, et al: Genome-wide miRNA expression profiling identifies miR-9-3 and miR-193a as targets for DNA methylation in non-small cell lung cancers. Clin Cancer Res 18: 1619-1629, 2012.

20. Iorio MV and Croce CM: microRNA involvement in human cancer. Carcinogenesis 33: 1126-1133, 2012.

21. Lehmann U, Hasemeier B, Christgen M, et al: Epigenetic inactivation of microRNA gene hsa-mir-9-1 in human breast cancer. J Pathol 214: 17-24, 2008.

22. Du Y, Liu Z, Gu L, et al: Characterization of human gastric carcinoma-related methylation of $9 \mathrm{miR} \mathrm{CpG}$ islands and repression of their expressions in vitro and in vivo. BMC Cancer 12: $249,2012$.

23. Lujambio A, Calin GA, Villanueva A, et al: A microRNA DNA methylation signature for human cancer metastasis. Proc Natl Acad Sci USA 105: 13556-13561, 2008.

24. Wan HY, Guo LM, Liu T, Liu M, Li X and Tang H: Regulation of the transcription factor NF-kappaB1 by microRNA-9 in human gastric adenocarcinoma. Mol Cancer 9: 16, 2010.

25. Hanahan D and Weinberg RA: The hallmarks of cancer. Cell 100: 57-70, 2000.

26. Jemal A, Siegel R, Ward E, Hao Y, Xu J and Thun MJ: Cancer statistics, 2009. CA Cancer J Clin 59: 225-249, 2009.

27. Vachon PH: Integrin signaling, cell survival, and anoikis: distinctions, differences, and differentiation. J Signal Transduct 2011: 738137, 2011.

28. Desiniotis A and Kyprianou N: Significance of talin in cancer progression and metastasis. Int Rev Cell Mol Biol 289: 117-147, 2011.

29. Fong YC, Liu SC, Huang CY, et al: Osteopontin increases lung cancer cells migration via activation of the alphavbeta3 integrin/ FAK/Akt and NF-kappaB-dependent pathway. Lung Cancer 64: 263-270, 2009.

30. Huang Y, Zhang X, Jiang W, et al: Discovery of serum biomarkers implicated in the onset and progression of serous ovarian cancer in a rat model using iTRAQ technique. Eur J Obstet Gynecol Reprod Biol 165: 96-103, 2012.

31. Vang R, Shih leM and Kurman RJ: Ovarian low-grade and highgrade serous carcinoma: pathogenesis, clinicopathologic and molecular biologic features, and diagnostic problems. Adv Anat Pathol 16: 267-282, 2009.

32. Critchley DR and Gingras AR: Talin at a glance. J Cell Sci 121: 1345-1347, 2008.

33. Osaki M, Oshimura M and Ito H: PI3K-Akt pathway: its functions and alterations in human cancer. Apoptosis 9: 667-676, 2004

34. Hwang YP, Yun HJ, Choi JH, et al: Suppression of EGF-induced tumor cell migration and matrix metalloproteinase-9 expression by capsaicin via the inhibition of EGFR-mediated FAK/Akt, PKC/Raf/ERK, p38 MAPK, and AP-1 signaling. Mol Nutr Food Res 55: 594-605, 2011.

35. Ren K, Jin H, Bian C, et al: MR-1 modulates proliferation and migration of human hepatoma HepG2 cells through myosin light chains-2 (MLC2)/focal adhesion kinase (FAK)/Akt signaling pathway. J Biol Chem 283: 35598-35605, 2008.

36. Sakamoto S, McCann RO, Dhir R and Kyprianou N: Talin1 promotes tumor invasion and metastasis via focal adhesion signaling and anoikis resistance. Cancer Res 70: 1885-1895, 2010 . 